\title{
Regional thalamic MRI as a marker of widespread cortical pathology and progressive frontotemporal involvement in amyotrophic lateral sclerosis
}

Sicong $\mathrm{Tu}^{1,2,3}$, Ricarda A.L. Menke ${ }^{2,3}$, Kevin Talbot ${ }^{3}$, Matthew C. Kiernan ${ }^{1}$, Martin R. Turner ${ }^{2,3}$

${ }^{1}$ Brain and Mind Centre, Sydney Medical School, University of Sydney, Sydney, Australia.

${ }^{2}$ Wellcome Centre for Integrative Neuroimaging, University of Oxford, UK.

${ }^{3}$ Nuffield Department of Clinical Neurosciences, University of Oxford, UK.

\section{Correspondence:}

Dr. Sicong Tu

Wellcome Centre for Integrative Neuroimaging

John Radcliffe Hospital

OX3 9DU

UK

sicong.tu@ndcn.ox.ac.uk

Word count: 3500

References: 40
Prof. Martin Turner

West Wing Level 6

John Radcliffe Hospital

OX3 9DU

UK

martin.turner@ndcn.ox.ac.uk 


\section{ABSTRACT}

\section{Background}

The thalamus is a major neural hub, with selective connections to virtually all cortical regions of the brain. The multi-system neurodegenerative syndrome amyotrophic lateral sclerosis (ALS) has pathogenic overlap with frontotemporal dementia, and objective in vivo markers of extramotor pathological spread are lacking. To better consider the role of the thalamus in neurodegeneration, the present study assessed the integrity of the thalamus and its connectivity to major cortical regions of the brain in a longitudinal manner.

\section{Methods}

Diffusion-based magnetic resonance imaging (MRI) tractography was utilised to parcellate the thalamus into distinct regions based on structural thalamo-cortical connectivity in 20 ALS patients, half of whom were scanned at two time points, and 31 matched controls scanned on a single occasion.

\section{Results}

At baseline, widespread diffusivity alterations in motor and extra-motor associated thalamic parcellations were detectable. Longitudinal decline selectively affected thalamic regions associated with frontal and temporal lobe connectivity. Diffusivity measures were significantly correlated with clinical measures of disease burden. Progression of functional disability, as indicated by change on the ALS functional rating scale, was associated with longitudinal change in mean diffusivity of the right frontal lobe thalamic parcellation $(r=0.59, \mathrm{p}=0.05)$.

\section{Conclusions}

Regional thalamic connectivity changes mirror the progressive frontotemporal cortical involvement associated with the motor functional decline in ALS. Longitudinal MRI thalamic parcellation has potential as a non-invasive surrogate marker of cortical dysfunction in ALS.

Keywords: amyotrophic lateral sclerosis; motor neuron disease; diffusion tensor imaging; tractography; thalamus 


\section{INTRODUCTION}

The thalamus is a major neural hub that receives and projects information to all cortical regions of the mammalian brain, where it has a critical role in sensory, motor and cognitive processes ${ }^{1}$. Although traditionally viewed as a relay structure, increasing evidence suggests that the thalamus plays an active role in modulating cortico-cortical information transfer with respect to behavioural context ${ }^{2}$. The cytoarchitecture of the thalamus itself is complex and unique, comprising numerous sub-nuclei with distinct projections to a diverse array of cortical and subcortical neural structures that can result in selective deficits of varying severity across a wide domain of brain functions when disrupted. These thalamic sub-nuclei can be parcellated in vivo, into distinct nuclei with divergent patterns of structural and functional connectivity using diffusion and functional MRI respectively ${ }^{34}$.

Across the neurodegenerative disease spectrum, amyotrophic lateral sclerosis (ALS) is characterised by the loss of upper motor neurons combined with loss of lower motor neurons arising from the brain stem and spinal cord anterior horns. ALS has a clinical, pathological, and genetic overlap with frontotemporal dementia (FTD) ${ }^{5}$. Further clinical heterogeneity includes site of initial symptom onset as well as rate of progression, with survival duration ranging from a few months to decades. Up to half of ALS patients may have demonstrable mild neuropsychological deficits ${ }^{6}$. Despite this clinical heterogeneity, nearly all cases of ALS are associated with a common underlying histopathology, namely deposition of a $43 \mathrm{kDa}$ phosphorylated transactive response DNA binding protein (pTDP-43) ${ }^{7}$. ALS is a syndrome that can be seen as a common endpoint of multiple upstream molecular pathways ${ }^{8}$. Stereotyped 
patterns of post mortem cerebral histological change have been identified ${ }^{9}$, lending support to wider evidence for a corticoefferent pathogenesis ${ }^{10}$, in which there might be propagation of protein misfolding through functionally connected brain networks ${ }^{11}$. While PTDP-43 aggregates appear to be concentrated in motor neurons, all cortical projection neurons that generate a long axon are posited to be vulnerable ${ }^{12}$. Thalamic involvement in ALS has been reported across in vivo neuroimaging platforms ${ }^{13-16}$, as well as post mortem histopathological studies ${ }^{717}$, although its role in the neurodegenerative process has not been articulated. As such, the present study tested the hypothesis that regional alterations in thalamic connectivity may serve as a holistic marker of the widespread cortical pathology and disease involvement in ALS.

\section{METHODS}

\section{Participants}

A group of ALS patients $(n=20)$ were selected from the longitudinal Oxford Study for Biomarkers in Motor Neurone Disease $($ BioMOx) cohort, matched in age $(\mathrm{p}=0.26)$ and education ( $p=0.13)$ with a group of healthy controls $(n=31)$. The patients were chosen from 65 potential MRI datasets according to a priori criteria: $<70$ years old at time of scan (to improve age-matching to controls and minimise independent age-related thalamic atrophy), apparently sporadic (no family history of ALS or FTD), classical ALS (not flail arm or other regionally limited phenotypes), no overt dementia, and a disease progression rate (DPR) $>0.4$ ALSFRS-R points per month. 
Ethical approval for this study was obtained from South Central Oxford Ethics Committee B (08/H0605/85). All participants provided written consent prior to assessment. The diagnosis of ALS was established by two experienced neurologists (MRT, KT). Patients were all presumed to be sporadic, given no family history of ALS or FTD. Patient clinical examinations were carried out at baseline and, for a subset $(n=11)$, again after an approximate 6-month interval (mean = 5.6 months, $\mathrm{SD}=0.8$ months). Disease duration was calculated from date of symptom onset to date of scan. Severity of functional disability was assessed at each clinical examination using the revised ALS functional rating scale (ALSFRS-R), a four-domain disability questionnaire with scores ranging from 0 to 48 , with a lower score reflecting greater disability ${ }^{18}$. DPR, as indicated by increasing disability, was calculated by dividing the change in the ALSFRS-R sum score at baseline and follow-up by the time between assessments. General cognitive function was assessed using the Addenbrooke’s Cognitive Examination-Revised (ACE-R), covering attention/orientation, memory, verbal fluency, language and visuospatial ability. ACE-R total score, as well as verbal fluency and memory recall sub-scores, whereby a higher score indicates better cognitive function.

\section{Image acquisition}

All participants were scanned at the Oxford Centre for Clinical Magnetic Resonance Research using a 3T Siemens Trio scanner with a 12-channel head coil on the day of the clinical examination. Whole-brain T1-weighted MRI scans were acquired using a magnetizationprepared rapid gradient echo (MPRAGE) sequence $(\mathrm{TR} / \mathrm{TE}=2040 / 4.7 \mathrm{~ms}$; flip angle = 8; $1 \mathrm{~mm}$ isotropic resolution). Whole-brain diffusion-weighted images were acquired using an echoplanar sequence $\left(60\right.$ isotropic directions; b-value $=1000 \mathrm{~s} / \mathrm{mm}^{2}$; echo time/repetition time $=$ 
94/10000 ms; 2 × 2 × 2 mm³ voxel size; 65 slices). Four additional b0 images without diffusion weighting were acquired. A field map was acquired using a gradient echo imaging sequence ( $2 \mathrm{x}$ 2 x 2 mm³ voxel size; 65 slices; echo time 1/echo time 2/repetition time $=$ 5.19/7.65/655 ms) to account for distortions present in diffusion-weighted data caused by field inhomogeneities.

\section{Volumetric and vertex analysis}

Each participant's thalami were segmented from their whole-brain T1-weighted MRI scan using FIRST $^{19}$ in FSL. Resulting segmentations were visually inspected for accuracy. For each participant, grey matter volume of the thalamus was then calculated after normalizing for interindividual differences in head size via the volumetric scaling factor derived from SIENAX ${ }^{20}$. Briefly, brain and skull images were extracted and affine-registered to MNI152 space, using the skull image to determine the registration scaling, to obtain a volumetric scaling factor accounting for the difference between the subject's image and standard space.

Focal morphological change along the surface of the thalami was then examined between patients and healthy controls using vertex analysis. Vertex analysis is a powerful statistical tool that provides complementary structural characteristics for specified structures of interest. Specifically, vertex locations from each participant were projected onto a deformable mesh surface of an average template as scalar values using FIRST in a mode of operation that assessed group differences on a per-vertex basis. Intergroup differences were assessed using permutation based non-parametric testing. Results with $\mathrm{p}<0.05$ were considered significant after clusterbased multiple comparison correction. 
Each individual's structural MRI scan was also processed in FreeSurfer 6.0 to generate cortical masks of interest to be used as target masks in subsequent tractography analyses. The standard cortical processing pipeline was carried out ${ }^{21}$, whereby scans were preprocessed and linearly aligned to the MNI305 average brain and segmented into tissue types. Images were aligned to a common spherical template surface using cortical folding-based co-registration patterns. After alignment, the cortex was partitioned on the basis of gyral and sulcal structures using an automated segmentation procedure. For each individual, left and right hemispheric cortical masks comprising the frontal, somatosensory, parietal, temporal, and occipital cortices, as defined by the Desikan-Killiany atlas ${ }^{22}$, were obtained, as well as Brodmann defined areas of premotor (BA6) and primary motor (BA4) cortices. All cortical masks of interest were transformed from spherical-space back to native space using FSL's affine registration tool FLIRT $^{23}$ and dilated to ensure masks reached white matter boundaries. Grey matter volumes of all cortical masks were extracted from FreeSurfer for each participant’s scan.

\section{Diffusion preprocessing and tractography}

All diffusion-weighted scans were corrected for head motion and eddy currents, then brainextracted to remove all non-brain voxels. Field map correction was performed with FUGUE to correct for field inhomogeneities and to unwarp scans. Following preprocessing, DTIFIT was used to apply a diffusion tensor model at each voxel, resulting in maps of three eigenvalues (L1, L2, L3), which allowed the calculation of fractional anisotropy (FA), mean diffusivity (MD), radial diffusivity (RD), and axial diffusivity (AD) maps for each participant's scan ${ }^{4}$. 
Using each individual's preprocessed diffusion-weighted image, scans were processed in FSL's BEDPOSTX to estimate the fibre orientation distribution function within each voxel for subsequent probabilistic tractography. Probabilistic tractography was performed independently within left and right hemispheres of the brain and in each subject's native structural space using PROBTRACKX, using the thalamus as a seed mask and the cortical structures obtained with FreeSurfer, as described above, as classification targets. Following tractography, seed-to-target information defining the connectivity between seed and target masks was used to perform a winner-takes-all clustering to assign each voxel within the thalamus to the target with which it showed the highest probability of connection ${ }^{4}$. Mean diffusion metrics (FA, MD, RD, AD) of each participant's left and right thalamic parcellations were extracted and compared between patients and controls.

For longitudinal analysis of 11 ALS patients, all 6 month follow-up structural and diffusion weighted scans were processed using the same protocol, and independent of baseline scans. Tractography based thalamic parcellation was performed in the same manner as at baseline, using cortical masks derived from follow-up structural patients scans processed in FreeSurfer. To ensure longitudinal diffusivity change was independent of volumetric differences of thalamic parcellations at baseline and follow-up, patient scans were transformed into a common 'halfway' space using SIENAX and diffusion metrics were extracted for each time point only where the parcellations intersect. Mean diffusion metrics of each patient's left and right thalamic parcellations at 6 month follow-up were extracted and compared to baseline. 


\section{Statistical analyses}

Statistical analyses comparing thalamic volume, cortical volume and diffusivity of thalamic parcellations between ALS and healthy controls were carried out using SPSS 21.0. The ShapiroWilk test was used to test normality. Total thalamic volume was compared between participant groups, and longitudinally within ALS patients using two-tailed independent samples t-test and paired samples t-test, respectively. Cortical mask volumes, and cluster size and DTI metrics averaged across thalamic parcellations relating to the frontal, premotor (BA6), primary motor (BA4), somatosensory, parietal, occipital and temporal cortices, were assessed using one-way multivariate analysis of variance (MANOVA) at baseline in patients and controls, followed by planned comparisons of each dependent variable between cohorts using Bonferroni correction. Longitudinal change within 11 ALS patients was analyzed using repeated measures MANOVA. Two-tailed Pearson's correlation was used to examine correlations between imaging metrics and clinical measures. Results were adjusted for multiple comparisons using Bonferroni correction. In all analyses p values $<0.05$ were considered to be significant.

\section{RESULTS}

\section{Patient data and demographics}

ALS and healthy control cohorts were well matched for age $(p=0.26)$ and years of education ( $p$ $=0.13$ ) (Table 1). Site of symptom onset in ALS patients varied, but was equally represented in upper $(\mathrm{n}=8)$ and lower $(\mathrm{n}=9)$ limb cases, and 3 bulbar onset cases. Patients were cognitively intact as indicated by overall performance on the ACE-R (mean $=93.6$, SD $=4.4$ ), scoring within 1 standard deviation of the normative population score for their age group (mean = 95.82, 
$\mathrm{SD}=3.8$ ), and above the high end cut off limit of 88 for suspected dementia. The ALS cohort recruited for the current study is representative of classical sporadic ALS. 
Table 1. Participant demographic characteristics and clinical profile. Mean and standard deviation.

\begin{tabular}{|c|c|c|c|}
\hline & $\begin{array}{c}\text { ALS } \\
(n=20)\end{array}$ & $\begin{array}{l}\text { Control } \\
(n=31)\end{array}$ & P-value \\
\hline $\operatorname{Sex}(M / F)$ & $14 \mathrm{M}, 6 \mathrm{~F}$ & $17 \mathrm{M}, 14 \mathrm{~F}$ & - \\
\hline Handedness (L/R) & $18 \mathrm{R}, 0 \mathrm{~L}$ & $25 \mathrm{R}, 3 \mathrm{~L}$ & - \\
\hline Age (y.o) & $57(10.7)$ & $53.1(12.1)$ & 0.26 \\
\hline Education (yrs) & $14(4.17)$ & $15.8(3.4)$ & 0.13 \\
\hline Disease Duration (months) & $20.7(9.2)$ & - & - \\
\hline ALSFRS-R (/48) & $34.6(5.2)$ & - & - \\
\hline Site of Symptom Onset & 3 Bulbar & - & - \\
\hline & 5 RUL, 3 LUL & & \\
\hline & 5 RLL, 4 LLL & & \\
\hline $\begin{array}{c}\text { Disease Progression Rate } \\
\text { (48 - ALSFRS-R / duration) }\end{array}$ & $0.75(0.3)$ & - & - \\
\hline \multicolumn{4}{|l|}{ ACE-R } \\
\hline Total (/100) & $93.6(4.4)$ & - & - \\
\hline VF-P Words (/7) & $5.4(1.5)$ & - & - \\
\hline VF-Animals (/7) & $6.3(1.1)$ & - & - \\
\hline Memory Recall (/7) & $5.6(1.5)$ & - & - \\
\hline
\end{tabular}

Note: Revised Amyotrophic Lateral Sclerosis Functional Rating Scale (ALSFRS-R); Addenbrooke's Cognitive Examination-Revised (ACE-R); Verbal fluency (VF); right upper limb (RUL); left upper limb (LUL); right lower limb (RLL); left lower limb (LLL). ACE-R data available from 12 ALS patients. 


\section{Volumetric and morphological thalamic change}

ALS patients showed reduced overall grey matter volume for the left $(\mathrm{p}=0.02)$ and right $(\mathrm{p}=$ 0.03) thalamus (Fig. 1A). This finding was further supported by complementary vertex analyses of the thalami, which indicated changes involving the medial surface of the left and right thalamus in ALS patients (Fig. 1B). No significant volumetric or morphological thalamic changes were observed longitudinally.

--------Insert Figure 1--------

\section{Structural connectivity based thalamic parcellation}

Each participant's left and right thalamus was reliably and consistently parcellated using tractography according to their ipsilateral structural connectivity to frontal, premotor (BA6), primary motor (BA4), somatosensory, parietal, temporal, and occipital cortices (Fig. 2). Grey matter volume of the cortical masks used as classification targets in tractography was compared between ALS patients and controls. A difference in volume of cortical masks was approaching significance based on cohort at baseline $(p=0.06)$. Follow-up comparisons for each cortical mask indicated ALS patients had significantly reduced bilateral grey matter volume of primary motor cortex (BA4), relative to healthy controls (all p < 0.01; Supplementary Table 1).

Longitudinally, there was a significant change in volume within subjects across cortical masks in ALS patients $(\mathrm{p}=0.03)$, with follow-up comparisons indicating significant bilateral grey matter volume reduction in the primary motor cortex (BA4) between baseline and 6 month follow-up (all p < 0.02). Cluster size of thalamic parcellations was also assessed at baseline and follow-up. No significant difference in cluster size was observed across thalamic parcellations between ALS 
patients and controls at baseline $(\mathrm{p}=0.61)$, or longitudinally within ALS patients $(\mathrm{p}=0.29$; Supplementary Table 2).

---------Insert Figure 2--------

Diffusion metrics indicated increased diffusion in thalamic parcellations associated with the left frontal lobe, bilateral premotor cortex, bilateral motor cortex, right somatosensory cortex, and bilateral parietal lobe, in ALS patients compared to healthy controls (all p < 0.05; Table 2). This pattern of increased diffusivity in ALS patients was consistent across diffusion metrics of MD, $\mathrm{RD}$ and $\mathrm{AD}$, and reflects greater diffusion occurring in these sub-thalamic regions as a result of reduced cellular integrity. No differences in FA were observed between ALS patients and healthy controls across thalamic parcellations. 
Table 2. Diffusion metrics of structural connectivity based thalamic parcellations in ALS patients and healthy controls. *Indicates significance at $\mathrm{p}<0.05$.

\begin{tabular}{|c|c|c|c|c|c|c|c|c|c|c|}
\hline \multirow[t]{2}{*}{ Thalamic Parcellation } & \multirow{2}{*}{$\begin{array}{c}\text { Hemis- } \\
\text { phere }\end{array}$} & \multirow[t]{2}{*}{ Group } & \multicolumn{2}{|c|}{ FA } & \multicolumn{2}{|c|}{ MD $\left(\times 10^{-3}\right)$} & \multicolumn{2}{|c|}{ RD $\left(\times 10^{-3}\right)$} & \multicolumn{2}{|c|}{$\mathrm{AD}\left(\mathrm{x} 10^{-3}\right)$} \\
\hline & & & Mean & SD & Mean & SD & Mean & SD & Mean & SD \\
\hline \multirow[t]{4}{*}{ Frontal Lobe } & \multirow[t]{2}{*}{$\mathrm{L}$} & ALS & 0.3 & 0.02 & $0.9 *$ & 0.07 & 1.02 & 0.07 & 1.16 & 0.07 \\
\hline & & Control & 0.31 & 0.02 & 0.85 & 0.07 & 0.97 & 0.07 & 1.11 & 0.07 \\
\hline & \multirow[t]{2}{*}{$\mathrm{R}$} & ALS & 0.3 & 0.02 & 0.93 & 0.09 & 1.04 & 0.09 & 1.18 & 0.09 \\
\hline & & Control & 0.3 & 0.03 & 0.89 & 0.11 & 1.01 & 0.11 & 1.15 & 0.11 \\
\hline \multirow[t]{4}{*}{ Premotor Cortex (BA6) } & \multirow[t]{2}{*}{$\mathrm{L}$} & ALS & 0.32 & 0.03 & $0.74 *$ & 0.06 & $0.85 *$ & 0.06 & $0.98 *$ & 0.07 \\
\hline & & Control & 0.34 & 0.04 & 0.69 & 0.04 & 0.81 & 0.04 & 0.93 & 0.05 \\
\hline & \multirow[t]{2}{*}{$\mathrm{R}$} & ALS & 0.33 & 0.03 & 0.76* & 0.06 & $0.87 *$ & 0.06 & 1.01* & 0.06 \\
\hline & & Control & 0.33 & 0.03 & 0.71 & 0.05 & 0.83 & 0.05 & 0.95 & 0.05 \\
\hline \multirow[t]{4}{*}{ Motor Cortex (BA4) } & \multirow[t]{2}{*}{$\mathrm{L}$} & ALS & 0.35 & 0.02 & $0.72^{*}$ & 0.03 & 0.83 & 0.04 & 1.01 & 0.06 \\
\hline & & Control & 0.37 & 0.04 & 0.7 & 0.03 & 0.81 & 0.03 & 0.99 & 0.05 \\
\hline & \multirow[t]{2}{*}{$\mathrm{R}$} & ALS & 0.35 & 0.04 & $0.75 *$ & 0.06 & $0.86 *$ & 0.06 & 1.04* & 0.06 \\
\hline & & Control & 0.35 & 0.04 & 0.71 & 0.03 & 0.82 & 0.03 & 0.98 & 0.04 \\
\hline \multirow{4}{*}{ Somatosensory Cortex } & \multirow[t]{2}{*}{$\mathrm{L}$} & ALS & 0.37 & 0.04 & 0.73 & 0.04 & 0.84 & 0.05 & 1.04 & 0.07 \\
\hline & & Control & 0.37 & 0.04 & 0.71 & 0.05 & 0.82 & 0.05 & 1.02 & 0.06 \\
\hline & \multirow[t]{2}{*}{$\mathrm{R}$} & ALS & 0.38 & 0.04 & $0.76 *$ & 0.07 & $0.88 *$ & 0.08 & 1.11* & 0.12 \\
\hline & & Control & 0.37 & 0.04 & 0.72 & 0.04 & 0.83 & 0.04 & 1.03 & 0.06 \\
\hline \multirow[t]{4}{*}{ Parietal Lobe } & \multirow[t]{2}{*}{$\mathrm{L}$} & ALS & 0.34 & 0.02 & $0.81 *$ & 0.08 & $0.93 *$ & 0.09 & 1.1* & 0.1 \\
\hline & & Control & 0.34 & 0.03 & 0.76 & 0.05 & 0.88 & 0.06 & 1.04 & 0.06 \\
\hline & \multirow[t]{2}{*}{$\mathrm{R}$} & ALS & 0.33 & 0.02 & 0.85 & 0.09 & $0.97 *$ & 0.1 & 1.14* & 0.1 \\
\hline & & Control & 0.32 & 0.03 & 0.81 & 0.06 & 0.93 & 0.07 & 1.09 & 0.07 \\
\hline \multirow[t]{4}{*}{ Temporal Lobe } & \multirow[t]{2}{*}{$\mathrm{L}$} & ALS & 0.26 & 0.03 & 1.18 & 0.11 & 1.3 & 0.11 & 1.47 & 0.11 \\
\hline & & Control & 0.27 & 0.03 & 1.11 & 0.12 & 1.22 & 0.12 & 1.4 & 0.13 \\
\hline & \multirow[t]{2}{*}{$\mathrm{R}$} & ALS & 0.25 & 0.03 & 1.25 & 0.19 & 1.37 & 0.2 & 1.54 & 0.21 \\
\hline & & Control & 0.26 & 0.03 & 1.2 & 0.16 & 1.31 & 0.16 & 1.48 & 0.16 \\
\hline \multirow[t]{4}{*}{ Occipital Lobe } & \multirow[t]{2}{*}{$\mathrm{L}$} & ALS & 0.32 & 0.04 & 0.83 & 0.15 & 0.95 & 0.15 & 1.1 & 0.16 \\
\hline & & Control & 0.32 & 0.04 & 0.81 & 0.12 & 0.92 & 0.13 & 1.08 & 0.16 \\
\hline & \multirow[t]{2}{*}{$\mathrm{R}$} & ALS & 0.31 & 0.03 & 0.86 & 0.14 & 0.97 & 0.15 & 1.13 & 0.17 \\
\hline & & Control & 0.32 & 0.06 & 0.84 & 0.15 & 0.96 & 0.15 & 1.12 & 0.17 \\
\hline
\end{tabular}


Longitudinal diffusivity change across thalamic parcellations in ALS patients was more selective (Table. 3). The right frontal lobe thalamic parcellation showed a significant increase in MD, RD and $\mathrm{AD}$ (all $\mathrm{p}<0.02$ ). The right and left temporal lobe thalamic parcellations also showed a significant increase in $\mathrm{MD}, \mathrm{RD}$ and $\mathrm{AD}($ all $\mathrm{p}<0.05)$. 
Table 3. Diffusion metrics of structural connectivity based thalamic parcellation in 11 ALS patients at baseline (T1) and 6-month follow-up(T2). *Indicates significant change between T1 and T2, p $<0.05$.

\begin{tabular}{|c|c|c|c|c|c|c|c|c|c|c|}
\hline \multirow[t]{2}{*}{ Thalamic Parcellation } & \multirow{2}{*}{$\begin{array}{c}\text { Hemis- } \\
\text { phere }\end{array}$} & \multirow{2}{*}{$\begin{array}{l}\text { Time- } \\
\text { point }\end{array}$} & \multicolumn{2}{|c|}{ FA } & \multicolumn{2}{|c|}{ MD $\left(\times 10^{-3}\right)$} & \multicolumn{2}{|c|}{$\mathrm{RD}\left(\times 10^{-3}\right)$} & \multicolumn{2}{|c|}{$\mathrm{AD}\left(\times 10^{-3}\right)$} \\
\hline & & & Mean & SD & Mean & $\mathrm{SD}$ & Mean & SD & Mean & $\mathrm{SD}$ \\
\hline \multirow[t]{4}{*}{ Frontal Lobe } & $\mathrm{L}$ & $\mathrm{T} 1$ & 0.3 & 0.02 & 0.85 & 0.07 & 0.97 & 0.08 & 1.1 & 0.07 \\
\hline & & $\mathrm{T} 2$ & 0.3 & 0.03 & 0.86 & 0.08 & 0.98 & 0.08 & 1.12 & 0.08 \\
\hline & $\mathrm{R}$ & $\mathrm{T} 1$ & 0.3 & 0.02 & $0.87 *$ & 0.07 & 0.99* & 0.07 & $1.12 *$ & 0.07 \\
\hline & & $\mathrm{T} 2$ & 0.3 & 0.03 & 0.92 & 0.1 & 1.04 & 0.1 & 1.18 & 0.1 \\
\hline \multirow[t]{4}{*}{ Premotor Cortex (BA6) } & $\mathrm{L}$ & $\mathrm{T} 1$ & 0.33 & 0.03 & 0.72 & 0.04 & 0.83 & 0.04 & 0.96 & 0.04 \\
\hline & & $\mathrm{T} 2$ & 0.35 & 0.03 & 0.7 & 0.03 & 0.82 & 0.03 & 0.96 & 0.03 \\
\hline & $\mathrm{R}$ & $\mathrm{T} 1$ & 0.35 & 0.03 & 0.72 & 0.04 & 0.84 & 0.04 & 0.99 & 0.05 \\
\hline & & $\mathrm{T} 2$ & 0.35 & 0.03 & 0.72 & 0.02 & 0.84 & 0.03 & 0.98 & 0.03 \\
\hline \multirow[t]{4}{*}{ Motor Cortex (BA4) } & $\mathrm{L}$ & $\mathrm{T} 1$ & 0.36 & 0.03 & 0.71 & 0.03 & 0.82 & 0.03 & 1 & 0.04 \\
\hline & & $\mathrm{T} 2$ & 0.38 & 0.02 & 0.7 & 0.02 & 0.82 & 0.02 & 1.01 & 0.03 \\
\hline & $\mathrm{R}$ & $\mathrm{T} 1$ & 0.36 & 0.03 & 0.72 & 0.02 & 0.83 & 0.02 & 1.02 & 0.04 \\
\hline & & $\mathrm{T} 2$ & 0.36 & 0.03 & 0.71 & 0.03 & 0.82 & 0.03 & 1 & 0.05 \\
\hline \multirow[t]{4}{*}{ Somatosensory Cortex } & $\mathrm{L}$ & $\mathrm{T} 1$ & 0.4 & 0.05 & 0.72 & 0.03 & 0.84 & 0.04 & 1.05 & 0.06 \\
\hline & & $\mathrm{T} 2$ & 0.4 & 0.06 & 0.72 & 0.03 & 0.84 & 0.04 & 1.06 & 0.07 \\
\hline & $\mathrm{R}$ & $\mathrm{T} 1$ & 0.4 & 0.06 & 0.73 & 0.04 & 0.84 & 0.04 & 1.07 & 0.06 \\
\hline & & $\mathrm{T} 2$ & 0.4 & 0.06 & 0.73 & 0.03 & 0.84 & 0.03 & 1.07 & 0.07 \\
\hline \multirow[t]{4}{*}{ Parietal Lobe } & $\mathrm{L}$ & $\mathrm{T} 1$ & 0.34 & 0.03 & 0.75 & 0.04 & 0.87 & 0.04 & 1.03 & 0.04 \\
\hline & & $\mathrm{T} 2$ & 0.36 & 0.04 & 0.75 & 0.04 & 0.88 & 0.04 & 1.05 & 0.04 \\
\hline & $\mathrm{R}$ & $\mathrm{T} 1$ & 0.34 & 0.02 & 0.78 & 0.03 & 0.89 & 0.04 & 1.06 & 0.05 \\
\hline & & $\mathrm{T} 2$ & 0.34 & 0.03 & 0.77 & 0.04 & 0.89 & 0.04 & 1.05 & 0.04 \\
\hline \multirow[t]{4}{*}{ Temporal Lobe } & $\mathrm{L}$ & $\mathrm{T} 1$ & 0.27 & 0.03 & $1.08 *$ & 0.13 & 1.19* & 0.14 & $1.37 *$ & 0.14 \\
\hline & & $\mathrm{T} 2$ & 0.26 & 0.03 & 1.18 & 0.15 & 1.29 & 0.15 & 1.48 & 0.15 \\
\hline & $\mathrm{R}$ & $\mathrm{T} 1$ & 0.27 & 0.02 & $1.15 *$ & 0.21 & $1.27 *$ & 0.22 & $1.45^{*}$ & 0.25 \\
\hline & & $\mathrm{T} 2$ & 0.26 & 0.04 & 1.21 & 0.21 & 1.33 & 0.22 & 1.5 & 0.23 \\
\hline \multirow[t]{4}{*}{ Occipital Lobe } & $\mathrm{L}$ & $\mathrm{T} 1$ & 0.35 & 0.07 & 0.8 & 0.2 & 0.92 & 0.2 & 1.09 & 0.21 \\
\hline & & $\mathrm{T} 2$ & 0.34 & 0.09 & 0.82 & 0.21 & 0.94 & 0.2 & 1.11 & 0.19 \\
\hline & $\mathrm{R}$ & $\mathrm{T} 1$ & 0.34 & 0.03 & 0.77 & 0.09 & 0.88 & 0.1 & 1.05 & 0.13 \\
\hline & & $\mathrm{T} 2$ & 0.35 & 0.05 & 0.77 & 0.09 & 0.89 & 0.1 & 1.06 & 0.11 \\
\hline
\end{tabular}




\section{Clinical correlates of thalamic integrity}

Volumetric measures of total grey matter volume of the thalami did not show any significant correlation with clinical measures of disease duration, absolute ALSFRS-R score, or rate of progression. Diffusion metrics however, showed a positive correlation with disease duration for the right premotor $(\mathrm{MD} r=0.48, \mathrm{p}=0.03$; $\mathrm{RD} \mathrm{r}=0.48, \mathrm{p}=0.03 ; \mathrm{AD} \mathrm{r}=0.51, \mathrm{p}=0.02)$, right primary motor $(\mathrm{MD} \mathrm{r}=0.6, \mathrm{p}<0.01$; $\mathrm{RD} \mathrm{r}=0.56, \mathrm{p}<0.01 ; \mathrm{AD} \mathrm{r}=0.45, \mathrm{p}=0.05)$, and right somatosensory (MD r = 0.61, $\mathrm{p}<0.01$; $\mathrm{RD} r=0.59, \mathrm{p}<0.01 ; \mathrm{AD} \mathrm{r}=0.48, \mathrm{p}=0.03)$ cortices respectively, associating increased diffusion with longer disease duration (Fig. 3A;

Supplementary Fig. 1A). Conversely, diffusion metrics showed a significant negative correlation with ALSFRS-R score for the right premotor $(\mathrm{MD} \mathrm{r}=-0.52, \mathrm{p}=0.02 ; \mathrm{RD} r=-0.53, \mathrm{p}=0.02$; $\mathrm{AD} \mathrm{r}=-0.59, \mathrm{p}<0.01)$, right primary motor $(\mathrm{MD} r=-0.57, \mathrm{p}<0.01 ; \mathrm{RD} r=-0.59, \mathrm{p}<0.01 ; \mathrm{AD}$ $\mathrm{r}=-0.53, \mathrm{p}=0.02)$, and right somatosensory $(\mathrm{r}=-0.6, \mathrm{p}<0.01 ; \mathrm{r}=-0.62, \mathrm{p}<0.01 ; \mathrm{r}=-0.61, \mathrm{p}<$ 0.01) cortices respectively, associating reduced diffusion with lower functional disability (Fig. 3B; Supplementary Fig. 1B). DPR, as indicated by change in ALSFRS-R at 6 month follow-up, showed a negative correlation with two diffusion metrics in the left temporal lobe $(\mathrm{RD} r=-0.43$, $\mathrm{p}=0.05 ; \mathrm{AD} r=-0.45, \mathrm{p}=0.05$ ) (Fig. 3C; Supplementary Fig. 1C). Relative to baseline, a positive correlation was detected between DPR and longitudinal change in MD of the right frontal lobe thalamic parcellation $(r=0.59, \mathrm{p}=0.05)$, but of sub-threshold significance with RD $(r=0.57, p=0.06)$ and $\mathrm{AD}(r=0.58, \mathrm{p}=0.06) . \mathrm{MD}, \mathrm{RD}$, and $\mathrm{AD}$ are all indicators of degree of diffusion, with increases indicative of reduced cellular integrity. The correlation between diffusion metrics and DPR suggest integrity of the left temporal and right frontal thalamic subregions are clinically tied to progressive functional disability in ALS patients. 


\section{DISCUSSION}

This study demonstrated widespread changes in thalamic cortical structural connectivity in ALS, involving extra-motor frontotemporal parcellations longitudinally, highlighting thalamic change as a holistic marker of disease burden in ALS. Some studies of thalamic grey matter change in ALS have reported reduction only in ALS-FTD ${ }^{24} 25$; in the $10 \%$ of ALS patients associated with a hexanucleotide repeat expansion (HRE) in C9orf72 24 26; or no detectable reduction ${ }^{27}$.

Diffusion MR studies in ALS consistently report markers of reduced thalamic cellular integrity ${ }^{28}$ 29. Thalamic abnormalities extend beyond conventional grey matter and diffusion change to altered functional connectivity ${ }^{28}$, increased iron deposition ${ }^{30}$, altered metabolism ${ }^{31}$, decreased texture features ${ }^{32}$, altered cerebral blood flow ${ }^{33}$, reduced motor task related activation ${ }^{34}$, and increased microglial activation ${ }^{35}$. Thus, despite the variability of detectable grey matter atrophy, thalamic abnormalities are a very robust finding consistent across a wider range of neuroimaging modalities (Table 4). 
Table 4. Summary of neuroimaging studies reporting thalamic abnormality in ALS.

\begin{tabular}{|c|c|c|c|}
\hline Participants & Methodology & Observed Thalamic Change & Reference \\
\hline 10 ALS; 14 Control & PK11195-PET & $\uparrow$ microglial activation & (Turner et al., 2004) \\
\hline 15 ALS; 12 Control & whole-brain voxel-wise DTI & $\downarrow \mathrm{FA}$ in right thalamus & (Sach et al., 2004) \\
\hline 10 ALS; 10 ALS-FTD; 22 Control & VBM & $\begin{array}{l}\text { ALS/ALS-FTD } \downarrow \text { grey matter in left thalamus } \\
\text { ALS-FTD } \downarrow \text { grey matter in left thalamus relative } \\
\text { to ALS }\end{array}$ & (Chang et al., 2005) \\
\hline 15 ALS; 25 Control & VBM, whole-brain voxel-wise DTI & $\begin{array}{l}\downarrow \text { total grey matter } \\
\downarrow \text { FA }\end{array}$ & (Thivard et al., 2007) \\
\hline 10 ALS; 12 ALS-bulbar; 22 Control & fMRI (vertical tongue movement) & $\downarrow$ task activation & (Mohammadi et al., 2009) \\
\hline 5 ALS; 5 ALS-dysphagia; 10 Control & $\begin{array}{l}\text { fMRI (swallowing paradigm), DTI } \\
\text { ROI analysis }\end{array}$ & $\begin{array}{l}\text { ALS } \uparrow \text { task activation of left thalamus relative to } \\
\text { ALS-dysphagia } \\
\downarrow \text { FA }\end{array}$ & (Li et al., 2009) \\
\hline 4 ALS-FUS & SPECT & $\downarrow$ blood flow (patient VI-4) & (Tateishi et al., 2010) \\
\hline 14 ALS; 17 Control & MRS & $\downarrow$ NAA, $\uparrow$ Cho & (Sharma et al., 2011) \\
\hline $\begin{array}{l}14 \text { ALS; } 11 \text { Control (longitudinal } 2 \\
\text { time-points) }\end{array}$ & CT perfusion & $\uparrow$ MTT at follow-up & (Murphy et al., 2012) \\
\hline 14 ALS; 12 Control & DTI ROI analysis & $\downarrow \mathrm{FA}, \uparrow \mathrm{MD}$ & (Sharma et al., 2013) \\
\hline 9 ALS C9+; 30 ALS C9-; 44 Control & TBSS & $\begin{array}{l}\text { ALS C9+ } \downarrow \text { AD relative to ALS C9- } \\
\text { ALS C9+ } \uparrow \mathrm{MD} / \mathrm{RD} / \mathrm{AD}\end{array}$ & (Bede et al., 2013) \\
\hline 36 ALS; 36 Control & VBM & $\downarrow$ total grey matter & (Menke et al., 2014) \\
\hline 24 ALS; 22 Control & DTI ROI analysis & $\uparrow \mathrm{MD}$ & (Barbagallo et al., 2014) \\
\hline $\begin{array}{l}30 \text { ALS; } 15 \text { ALS C9+; } 12 \text { ALS-FTD; } \\
40 \text { Control }\end{array}$ & FDG-PET & ALS C9+ $\downarrow$ metabolism relative to ALS/Control & (Cistaro et al., 2014) \\
\hline 11 ALS C9+; 59 ALS C9- & FDG-PET & ALS C9+ $\downarrow$ metabolism relative to ALS C9- & (van Laere et al., 2014) \\
\hline $\begin{array}{l}18 \text { ALS CI; } 42 \text { ALS NCI; } 7 \text { ALS- } \\
\text { FTD; } 39 \text { Control }\end{array}$ & VBM, vertex analysis, ROI analysis & ALS-FTD $\downarrow$ total grey matter/morphology & (Machts et al., 2015) \\
\hline 32 ALS; 32 Control & $\begin{array}{l}\text { FreeSurfer, whole-brain voxel-based } \\
\text { texture analysis }\end{array}$ & $\begin{array}{l}\downarrow \text { texture features } \\
\downarrow \text { total grey matter of right thalamus }\end{array}$ & (de Albuquerque et al., 2016) \\
\hline 19 ALS; 20 Control & $\begin{array}{l}\text { Whole-brain voxel-based texture } \\
\text { analysis }\end{array}$ & $\downarrow$ texture features & (Maani et al., 2016) \\
\hline 14 ALS C9+; 156 ALS C9- & ROI analysis & ALS C9+ $\downarrow$ total grey matter & (Westeneng et al., 2016) \\
\hline 38 ALS; 35 Control & Tractography-based parcellation & $\downarrow$ FA of premotor/motor region & (Zhang et al., 2017) \\
\hline 37 ALS; 37 Control & FDG-PET & $\downarrow$ metabolism & (Bubour et al., 2017) \\
\hline 20 ALS; 21 Control & Whole-brain graph analysis & $\downarrow$ nodal efficiency of right thalamus & (Xu et al., 2017) \\
\hline $\begin{array}{l}12 \text { ALS; } 38 \text { FTD; } 28 \text { Control (post- } \\
\text { mortem) }\end{array}$ & Iron deposition & $\uparrow$ iron deposition & (de Reuck et al., 2017) \\
\hline
\end{tabular}


16 ALS (longitudinal, 5 time-points)

36 ALS; 14 ALS-FTD C9+; 12 ALS-

FTD C9-; 50 Control
Progressive $\downarrow$ total grey matter/morphology

Progressive $\downarrow$ FA of right thalamus

Progressive $\uparrow \mathrm{MD} / \mathrm{RD} / \mathrm{AD}$

Progressive $\downarrow$ rs-fMRI connectivity

ALS-FTD C9+/- $\downarrow$ total grey matter

ALS-FTD C $+\downarrow$ grey matter of

motor/somatosensory region
(Menke et al., 2018)

(Bede et al., 2018)

Note: C9orf72 (C9); Cognitive impairment (CI); No cognitive impairment (NCI); Fused in sarcoma (FUS); Voxel-based morphometry (VBM); Region of

interest (ROI); Tract-based spatial statistics (TBSS); Independent components analysis (ICA); Resting state functional MRI (rs-fMRI); Fluoro-2-deoxy-D-

glucose positron emission tomography (FDG-PET); Computed tomography (CT); Mean transit time (MTT); Magnetic resonance spectroscopy (MRS); N-

acetylaspartate (NAA); Choline (Cho); Single-photon emission computed tomography (SPECT). Reported observed thalamic change was bilateral or laterality

was unclear, and relative to healthy controls, unless otherwise stated. Full reference list provided in Supplementary Table 3. 


\section{Thalamic grey matter changes}

Our cohort of ALS patients showed a significant bilateral reduction in overall volume of the thalamus at baseline, but no significant longitudinal change. Complementary vertex analysis supported this finding and also demonstrated significant bilateral boundary reduction along the midline of the thalamus, a region typically associated with connectivity to the frontal lobe. Factors that have been associated with more generalized cerebral atrophy in ALS are the presence of cognitive impairment ${ }^{36}$, comorbid FTD ${ }^{37}$, and the C9orf72 HRE ${ }^{26}$. It is possible that one or two of our presumed sporadic ALS patient cohort were carriers of the C9orf72 HRE, though highly unlikely to be a significant confound if so. Likewise, while the sub-optimal cognitive assessment used in this study predated tools with more sensitivity to ALS-related cognitive impairment and behavioural change such as the ECAS ${ }^{38}$, none of this study's participants showed features indicative of dementia.

\section{Diffusion changes in thalamic parcellations}

At baseline, widespread increased apparent diffusion was detected in parcellated thalamic regions associated with structural connectivity to frontal, premotor, primary motor, somatosensory, and parietal cortices. Mathematically, the lack of difference in FA across thalamic parcellations observed here is likely the result of $\mathrm{MD}, \mathrm{RD}$ and $\mathrm{AD}$ all showing a similar pattern of increased diffusivity in patients maintaining a similar ratio. This might reflect a lack of preferential diffusivity change along specific radiating directions due to the lack of isolated bundles of parallel fibres within the grey matter of the thalamus. 
Longitudinal diffusivity change was considerably more selective, affecting only the right frontal lobe and bilateral temporal lobe thalamic areas. The observed thalamic abnormalities were also found to relate to disease duration, functional disability rating, and progressive functional decline. Disease duration and ALSFRS-R score significantly correlated with degree of diffusivity in the premotor, primary motor and somatosensory parcellated regions of the right thalamus at baseline. This is consistent with previous studies that have linked disease duration and ALSFRSR score with motor related integrity in ALS ${ }^{29}$. Our finding of negative correlation between DPR and diffusivity of the baseline left temporal lobe thalamic parcellation, and positive correlation between DPR and longitudinal change in diffusivity of the right frontal lobe thalamic parcellations requires consideration. One might infer that cellular integrity of left temporothalamic connectivity and the increased longitudinal decline in cellular integrity of the right fronto-thalamic connectivity at baseline are associated with rate of progression in ALS. Rather than integrity of motor related thalamic connectivity, rate of disease progression might then be linked to imaging markers of frontotemporal involvement, even in ALS without overt cognitive impairment.

The interpretation of observed diffusivity change in the thalamus remains conservative, with limited assumptions on the cellular impact of diffusion alterations in grey matter. This remains important when dealing with iron rich deep grey matter structures in an elderly participant population. Not only does level of ferritin-bound iron impact MR signal, including diffusivity metrics, but levels naturally elevate as a consequence of ageing ${ }^{39}$. Of relevance, this confound has been reported to have little effect on the diffusivity of the thalamus, compared to the basal ganglia ${ }^{39}$. The importance of a demographically well-matched patient and control cohort for 
deep grey matter investigation remains critical, and undoubtedly there remains a major issue of clinical heterogeneity inherent to single centre studies of limited size, which multi-centre initiatives are looking to address. This study only considered the thalamus, focused as it was on a potential surrogate of widespread cortical pathology. The structural and functional relationship of the broader basal ganglia (e.g. caudate) to thalamic changes in ALS is an important concept for future study. Similarly, this study was not powered to explore potential hemispheric lateralization of thalamic pathology, which remains more broadly unanswered in the ALS neuroimaging literature. Pooling of larger numbers of clinically homogeneous cases through multi-centre ALS cohorts arising from the Neuroimaging Society in ALS ${ }^{40}$, offers real potential for addressing these more intricate clinicopathological correlates.

In summary, thalamic involvement is a consistent feature of the ALS disease signature. This study has demonstrated the utility of diffusion-based thalamic parcellation as a non-invasive marker of disease progression in ALS.

\section{ACKNOWLEDGEMENTS}

The authors would like to thank all the study participants for their efforts and enthusiasm for clinical research. The Oxford MND Centre (MRT, KT) receives funding from the Motor Neurone Disease Association. ST is funded by the Australian National Health and Medical Research Council CJ Martin Early Career Fellowship (APP1121859). MCK was supported by the Australian National Health and Medical Research Council Program Grant (Forefront 
\#1037746). MRT is funded by the Medical Research Council and Motor Neurone Disease Association Lady Edith Wolfson Senior Clinical Fellowship (MR/K01014X/1). 


\section{FIGURE LEGENDS}

Figure 1. Structural integrity of the thalamus in ALS patients compared to healthy controls. (a) Significant bilateral reduction in total thalamic volume in ALS compared to control, corrected for intracranial volume. * Indicates significance at $\mathrm{p}<0.05$ (b) 3D visualization of significant reduction along the medial surface of the thalamus bilaterally in ALS compared to control. Thalamus is shown in red, significant clusters shown in blue. Clusters are significant at $\mathrm{p}<0.05$, corrected for multiple comparisons.

Figure 2. Group average structural connectivity based parcellation map of the thalami to distinct cortical regions using a winner-takes-all tractography approach. Parcellations were thresholded at $30 \%$, and presented on the MNI152 standard brain.

Figure 3. Significant clinical correlates of disease duration, ALSFRS-R score, and DPR, with baseline diffusivity of thalamic parcellations in ALS patients. All correlations significant at $\mathrm{p}<$ 0.05, dotted lines indicate 95\% confidence of linear model fit. Same pattern of significant correlations found between clinical correlates of disease duration and ALSFRS-R with mean and radial diffusivity, shown in supplementary. 


\section{REFERENCES}

1. Sommer MA. The role of the thalamus in motor control. Current Opinion in Neurobiology 2003;13(6):663-70. doi: https://doi.org/10.1016/j.conb.2003.10.014

2. Sherman SM. Thalamus plays a central role in ongoing cortical functioning. Nat Neurosci 2016;19(4):533-41. doi: 10.1038/nn.4269

3. Ji B, Li Z, Li K, et al. Dynamic thalamus parcellation from resting-state fMRI data. Hum Brain Mapp 2016;37(3):954-67. doi: 10.1002/hbm.23079

4. Behrens TEJ, Johansen-Berg $\mathrm{H}$, Woolrich MW, et al. Non-invasive mapping of connections between human thalamus and cortex using diffusion imaging. Nature Neuroscience 2003;6(7):750-57. doi: $10.1038 / \mathrm{nn} 1075$

5. Burrell JR, Halliday GM, Kril JJ, et al. The frontotemporal dementia-motor neuron disease continuum. Lancet (London, England) 2016;388(10047):919-31. doi: 10.1016/s0140-6736(16)00737-6 [published Online First: 2016/03/19]

6. Strong MJ, Abrahams S, Goldstein LH, et al. Amyotrophic lateral sclerosis - frontotemporal spectrum disorder (ALS-FTSD): Revised diagnostic criteria. Amyotrophic lateral sclerosis \& frontotemporal degeneration 2017;18(3-4):153-74. doi: 10.1080/21678421.2016.1267768 [published Online First: 2017/01/06]

7. Neumann M, Sampathu DM, Kwong LK, et al. Ubiquitinated TDP-43 in frontotemporal lobar degeneration and amyotrophic lateral sclerosis. Science 2006;314(5796):130-3. doi: 10.1126/science. 1134108

8. Turner MR, Swash M. The expanding syndrome of amyotrophic lateral sclerosis: a clinical and molecular odyssey. Journal of neurology, neurosurgery, and psychiatry 2015;86(6):667-73. doi: 10.1136/jnnp-2014-308946 [published Online First: 2015/02/04]

9. Brettschneider J, Del Tredici K, Toledo JB, et al. Stages of pTDP-43 pathology in amyotrophic lateral sclerosis. Annals of neurology 2013;74(1):20-38. doi: 10.1002/ana.23937 [published Online First: 2013/05/21]

10. Eisen A, Braak H, Del Tredici K, et al. Cortical influences drive amyotrophic lateral sclerosis. J Neurol Neurosurg Psychiatry 2017 doi: 10.1136/jnnp-2017-315573

11. Seeley WW, Crawford RK, Zhou J, et al. Neurodegenerative diseases target large-scale human brain networks. Neuron 2009;62(1):42-52.

12. Braak H, Brettschneider J, Ludolph AC, et al. Amyotrophic lateral sclerosis--a model of corticofugal axonal spread. Nat Rev Neurol 2013;9(12):708-14. doi: 10.1038/nrneurol.2013.221

13. Turner MR, Cagnin A, Turkheimer FE, et al. Evidence of widespread cerebral microglial activation in amyotrophic lateral sclerosis: an [(11)C](R)-PK11195 positron emission tomography study. NeurobiolDis 2004;15(3):601-09.

14. Chang JL, Lomen-Hoerth C, Murphy J, et al. A voxel-based morphometry study of patterns of brain atrophy in ALS and ALS/FTLD. Neurology 2005;65(1):75-80.

15. Bede $P$, Elamin $M, B y r n e ~ S$, et al. Basal ganglia involvement in amyotrophic lateral sclerosis. Neurology 2013;81(24):2107-15. doi: 10.1212/01.wnl.0000437313.80913.2c [published Online First: 2013/11/12]

16. Menke RA, Korner S, Filippini N, et al. Widespread grey matter pathology dominates the longitudinal cerebral MRI and clinical landscape of amyotrophic lateral sclerosis. Brain 2014;137(Pt 9):254655. doi: 10.1093/brain/awu162

17. Smith MC. Nerve fibre degeneration in the brain in amyotrophic lateral sclerosis. JNeurolNeurosurgPsychiatry 1960;23:269-82. 
18. Cedarbaum JM, Stambler N, Malta E, et al. The ALSFRS-R: a revised ALS functional rating scale that incorporates assessments of respiratory function. BDNF ALS Study Group (Phase III). J Neurol Sci 1999;169(1-2):13-21.

19. Patenaude B, Smith SM, Kennedy DN, et al. A Bayesian model of shape and appearance for subcortical brain segmentation. Neuroimage 2011;56(3):907-22. doi: 10.1016/j.neuroimage.2011.02.046

20. Smith SM, Zhang Y, Jenkinson M, et al. Accurate, robust, and automated longitudinal and crosssectional brain change analysis. Neuroimage 2002;17(1):479-89.

21. Fischl B, Sereno MI, Dale AM. Cortical surface-based analysis. II: Inflation, flattening, and a surfacebased coordinate system. Neuroimage 1999;9(2):195-207. doi: 10.1006/nimg.1998.0396

22. Desikan RS, Segonne F, Fischl B, et al. An automated labeling system for subdividing the human cerebral cortex on MRI scans into gyral based regions of interest. Neuroimage 2006;31(3):96880. doi: 10.1016/j.neuroimage.2006.01.021

23. Jenkinson M, Smith S. A global optimisation method for robust affine registration of brain images. Med Image Anal 2001;5(2):143-56.

24. Bede $P$, Omer $T$, Finegan $E$, et al. Connectivity-based characterisation of subcortical grey matter pathology in frontotemporal dementia and ALS: a multimodal neuroimaging study. Brain Imaging Behav 2018 doi: 10.1007/s11682-018-9837-9

25. Machts J, Loewe K, Kaufmann J, et al. Basal ganglia pathology in ALS is associated with neuropsychological deficits. Neurology 2015;85(15):1301-09. doi: 10.1212/Wnl.0000000000002017

26. Westeneng HJ, Walhout $R$, Straathof $M$, et al. Widespread structural brain involvement in ALS is not limited to the C9orf72 repeat expansion. J Neurol Neurosurg Psychiatry 2016;87(12):1354-60. doi: 10.1136/jnnp-2016-313959

27. Bueno APA, Pinaya WHL, Moura LM, et al. Structural and functional papez circuit integrity in amyotrophic lateral sclerosis. Brain Imaging Behav 2018 doi: 10.1007/s11682-018-9825-0

28. Menke RAL, Proudfoot M, Talbot K, et al. The two-year progression of structural and functional cerebral MRI in amyotrophic lateral sclerosis. Neuroimage Clin 2018;17:953-61. doi: 10.1016/j.nicl.2017.12.025

29. Zhang JQ, Ji B, Zhou CY, et al. Differential Impairment of Thalamocortical Structural Connectivity in Amyotrophic Lateral Sclerosis. CNS Neurosci Ther 2017;23(2):155-61. doi: 10.1111/cns.12658

30. De Reuck J, Devos D, Moreau C, et al. Topographic distribution of brain iron deposition and small cerebrovascular lesions in amyotrophic lateral sclerosis and in frontotemporal lobar degeneration: a post-mortem 7.0-tesla magnetic resonance imaging study with neuropathological correlates. Acta Neurologica Belgica 2017;117(4):873-78. doi: 10.1007/s13760-017-0832-5

31. Buhour MS, Doidy F, Mondou A, et al. Voxel-based mapping of grey matter volume and glucose metabolism profiles in amyotrophic lateral sclerosis. EJNMMI Res 2017;7(1):21. doi: 10.1186/s13550-017-0267-2

32. de Albuquerque M, Anjos LG, Maia Tavares de Andrade H, et al. MRI Texture Analysis Reveals Deep Gray Nuclei Damage in Amyotrophic Lateral Sclerosis. J Neuroimaging 2016;26(2):201-6. doi: 10.1111/jon.12262

33. Murphy MJ, Grace GM, Tartaglia MC, et al. Widespread cerebral haemodynamics disturbances occur early in amyotrophic lateral sclerosis. Amyotroph Lateral Scler 2012;13(2):202-9. doi: 10.3109/17482968.2011.625569

34. Mohammadi B, Kollewe K, Samii A, et al. Decreased brain activation to tongue movements in amyotrophic lateral sclerosis with bulbar involvement but not Kennedy syndrome. J Neurol 2009;256(8):1263-9. doi: 10.1007/s00415-009-5112-8 
35. Turner MR, Cagnin A, Turkheimer FE, et al. Evidence of widespread cerebral microglial activation in amyotrophic lateral sclerosis: an [11C](R)-PK11195 positron emission tomography study. Neurobiol Dis 2004;15(3):601-9. doi: 10.1016/j.nbd.2003.12.012

36. Mioshi E, Lillo $P$, Yew B, et al. Cortical atrophy in ALS is critically associated with neuropsychiatric and cognitive changes. Neurology 2013;80(12):1117-23. doi: 10.1212/WNL.0b013e31828869da

37. Ambikairajah A, Devenney E, Flanagan E, et al. A visual MRI atrophy rating scale for the amyotrophic lateral sclerosis-frontotemporal dementia continuum. Amyotroph Lateral Scler Frontotemporal Degener 2014;15(3-4):226-34. doi: 10.3109/21678421.2014.880180

38. Niven E, Newton J, Foley J, et al. Validation of the Edinburgh Cognitive and Behavioural Amyotrophic Lateral Sclerosis Screen (ECAS): A cognitive tool for motor disorders. Amyotrophic lateral sclerosis \& frontotemporal degeneration 2015;16(3-4):172-9. doi: 10.3109/21678421.2015.1030430 [published Online First: 2015/05/15]

39. Pfefferbaum A, Adalsteinsson E, Rohlfing $T$, et al. Diffusion tensor imaging of deep gray matter brain structures: effects of age and iron concentration. Neurobiol Aging 2010;31(3):482-93. doi: 10.1016/j.neurobiolaging.2008.04.013

40. Turner MR, Grosskreutz J, Kassubek J, et al. Towards a neuroimaging biomarker for amyotrophic lateral sclerosis. Lancet Neurol 2011;10(5):400-3. doi: 10.1016/S1474-4422(11)70049-7 